\title{
Bone and Mineral Metabolism
}

\author{
Dov Tiosano \\ Meyer Children's Hospital, Haifa, Israel
}

\section{New genes and concepts}

\section{Leptin regulation of bone resorption by the sympathetic nervous system and CART}

\author{
Elefteriou F, Ahn JD, Takeda S, Starbuck M, Yang X, Liu X, Kondo H, Richards WG, Bannon TW, Noda M, \\ Clement K, Vaisse C, Karsenty G \\ Department of Molecular and Human Genetics, Baylor College of Medicine, Houston, Tex., USA \\ Nature 2005;434:514-520
}

Background: Leptin controls bone formation through sympathetic signaling via $\beta_{2}$-adrenergic receptors (Adrb2) present on osteoblasts. The aim of the study was to investigate bone metabolism in Adrb2 null mice.

Methods: Osteoblast and osteoclast differentiation and maturation were studied in Adrb2 null mice. The cellular cascade that lies between Adrb2 receptors and Rankl expression was investigated. The role of low leptin 'tone' and low estrogen levels on bone metabolism were studied in Adrb2 $2^{-1-}$ ovariectomized mice, as compared to ob/ob mice that present low leptin 'tone' and hypogonadotrophic hypogonadism. Results: By analyzing Adrb2-deficient mice, the authors show that the sympathetic nervous system favors bone resorption by increasing expression in osteoblast progenitor cells of the osteoclast differentiation factor Rankl. This sympathetic function requires phosphorylation (by protein kinase A) of ATF4, a cellspecific CREB-related transcription factor essential for osteoblast differentiation and function. Bone resorption is not increased in gonadectomized Adrb2-deficient mice. This contrasts sharply with the increase in bone resorption characterizing another hypogonadal mouse with low sympathetic tone, the ob/ob mouse. This discrepancy is explained, in part, by the fact that CART ('cocaine amphetamineregulated transcript'), a neuropeptide whose expression is controlled by leptin and nearly abolished in ob/ob mice, inhibits bone resorption by modulating Rankl expression.

Conclusion: This study establishes that leptin-regulated neural pathways control both aspects of bone remodeling, and demonstrates that integrity of sympathetic signaling is necessary for the increase in bone resorption caused by gonadal failure.

This study adds to our knowledge another tier concerning the connection between bone density, adipose tissue and the integrity of the central nervous system. It has been shown that leptin has a role in the regulation of bone mineral density. This regulation is mediated via the adrenergic system through the adrenergic $\beta_{2}$-receptor (Adrb2). In this study the authors show that in Adrb2 null mice the bone mineral density is higher than in the wt and the ob/ob (leptin-deficient) mice. Transplantation of $w t$ bone mineral cells (BMC) into $\gamma$-irradiated Adrb2 ${ }^{-1-}$ mice normalized bone formation parameters, and conversely, transplantation of $\mathrm{Adrb2}^{-1-} \mathrm{BMC}$ into $\gamma$-irradiated wt mice significantly increased bone formation parameters. In Adrb2 ${ }^{-1-}$ mice Rankl expression was reduced. Gonadectomized Adrb2 ${ }^{-1-}$ mice were expected to have a similar phenotype to the ob/ob mice that exhibit low sympathetic tone, and are hypogonadic. Surprisingly, in gonadectomized Adrb2 ${ }^{-l^{-}}$bone mass did not decrease.

The authors hypothesized that expression of genes regulating bone resorption is disturbed in ob/ob mice but not in gonadectomized Adrb2 ${ }^{-1-}$ mice. The authors focused on genes whose expression is regulated by leptin, and whose inactivation does not affect appetite or fertility. By this approach, CART ('cocaine amphetamine-regulated transcript') was found. CART encodes a neuropeptide whose expression is enhanced in the brain by leptin. In ob/ob mice its expression is low, while in Adrb2 $2^{-I-}$ mice its expression is normal. CART ${ }^{-1-}$ mice have a low bone mass. They have normal osteoblast numbers, and on the other hand, their osteoclast surface and number is doubled. Rankl expression is 
upregulated in $\mathrm{CART}^{-1-}$ bones, suggesting that CART modulated bone resorption by inhibiting Rankl signaling.

An example for the correlation between CART and bone mineral density can be found in the

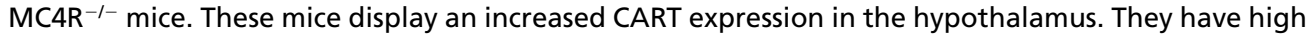
bone mass with a marked decrease in osteoclast number.

This study highlights the significance of the nervous system in its entirety in relation to bone metabolism and shows that estrogen deficiency is not an independent cause for the decline of bone mass. This study raises the question regarding the role of CART in the physiological process of osteoporosis in the elderly and as a factor during chronic illnesses that affects bone metabolism. However, there is still a missing part in the puzzle, since the molecular basis for CART regulation of bone resorption is still unclear. No specific CART receptor was identified in the bone.

\section{Circulating osteoblasts - where do they go?}

\section{Circulating osteoblast-lineage cells in humans}

Eghbali-Fatourechi GZ, Lamsam J, Fraser D, Nagel D, Riggs BL, Khosla S

Endocrine Research Unit, Mayo Clinic College of Medicine, Rochester, Minn., USA

N Engl J Med 2005;352:1959-1966

Background: Current evidence suggests that only a minuscule number of osteoblast-lineage cells are present in peripheral blood. The authors hypothesized that the concentration of these cells is elevated during times of increased bone formation, such as during pubertal growth.

Methods: Flow cytometry, with antibodies to bone-specific proteins, was used to identify circulating osteoblast-lineage cells in 11 adolescent males and 11 adult males (mean [ \pm SD] age, $14.5 \pm 0.7$ vs. $37.7 \pm 7.6$ years). Gene expression and in vitro and in vivo bone-forming assays were used to establish the osteoblastic lineage of sorted cells.

Results: Cells positive for osteocalcin and cells positive for bone-specific alkaline phosphatase were detected in the peripheral blood of adult subjects (1-2\% of mononuclear cells). There were more than 5 times as many cells positive for osteocalcin in the circulation of adolescent boys as compared with adult subjects $(p<0.001)$. The percentage of cells positive for osteocalcin correlated with markers of bone formation. Sorted osteocalcin-positive cells expressed osteoblastic genes, formed mineralized nodules in vitro, and formed bone in an in vivo transplantation assay. Increased values were also found in 3 adults with recent fractures.

Conclusions: Osteoblast-lineage cells circulate in physiologically significant numbers, correlate with markers of bone formation, and are markedly higher during pubertal growth.

This study provides clear evidence that osteoblast-lineage (osteocalcin-producing) cells are present in the human circulation in significant numbers, and that the percentage of circulating osteocalcin-positive cells is higher during the adolescent growth spurt. This is associated with a marked stimulation of bone formation as well as during bone healing after fractures. This observation brings hope that circulating osteoblast-lineage cells might also have therapeutic potential. Developing a method to harvest these cells from peripheral blood, expand them in culture, and subsequently implant them into sites of impaired bone healing might lead to new approaches to bone regeneration.

\section{Bone measurements Important observations for clinical practice}

Dual energy $x$-ray absorptiometry (DEXA) is based on the method of $x$-ray spectrophotometry developed in the 1970s. The technique was originally developed for use in adults. The attempt to use this technique for children raises some problems since children are not just small adults. The size and the 
shape of children's bones differ from mature bones as their bones grow, their body weight changes, and they develop sexually.

Technically, DEXA calculates a two-dimensional bone projectional area and the output are expressed as an 'area density' (BMD area). In order to correct the values for lumbar or femoral BMD to the estimated bone size, mathematical models are used. Several approaches are proposed to correct for the impact of body size, body weight, and Tanner stage of puberty. One approach is to calculate a volumetric or bone mineral apparent density (BMAD) using the bone area and average width of the lumbar vertebrae to produce a measure of bone volume [1]. Although such an approach makes a clear adjustment for the effect of bone size, it fails to take into account all issues concerning body size resulting in an over-approximation of bone mass for children with large wide bones. Normative data involving adjustments of BMC for projected bone area, body height, weight and Tanner stage of puberty using a regression model as recommended by Prentice et al. [2], were published by Warner et al. [3] and Ellis et al. [4]. A third approach proposed by Molgaard et al. [5] uses a three-stage algorithm to evaluate whole-body BMC, which considers height for age, BA for height and BMC for BA. These three steps were suggested to correspond to three different reasons for reduced bone mass, short bones, narrow bones and light bones.

In contrast to DEXA, CT can determine the volumetric bone mineral density as it is measured and not calculated. Wren et al. compared these two methods in children and adolescents in order to define the best way to estimate vertebral bone density.

\section{Bone acquisition in healthy children and adolescents: comparisons of dual-energy $x$-ray absorptiometry and computed tomography measures}

Wren TA, Liu X, Pitukcheewanont P, Gilsanz V

Department of Radiology, Los Angeles, Calif., USA

vgilsanz@chla.nsc.edu

J Clin Endocrinol Metab 2005;90:1925-1928

Background: The aim of this study was to determine the best method for optimizing pediatric bone measurements using DXA.

Methods: 64 boys and 60 healthy girls ages 6-17 years were enrolled in the study. Height, weight, body mass index, skeletal age, and Tanner stage of sexual development were determined. DXA of the lumbar vertebrae measured bone mineral content (BMC, in grams) and areal bone mineral density (aBMD). Geometric corrections were used to calculate volumetric bone mineral densities (vBMD). Computed tomography $(\mathrm{CT})$ imaging was performed to measure volumetric bone density (vBD) and vertebral volume $(\mathrm{Vol})$ in order to calculate CT-BMC $=\mathrm{vBD} \cdot$ Vol. Linear regression was used to compare DXA$\mathrm{BMC}$ vs. CT-BMC. Multiple regression including the anthropometric and developmental parameters were also performed.

Results: DXA and CT BMC were highly correlated. However, DXA aBMD correlated more strongly with CT Vol than with CT density. The calculation of DXA volumetric densities only slightly improved the density correlations. The correlations for density were particularly poor for subjects in Tanner stages 1-3. Multiple regression accounting for the anthropometric and developmental parameters greatly improved the agreement between the DXA and CT densities.

Conclusion: These results suggest that DXA BMC is a more accurate and reliable measure than DXA BMD for assessing bone acquisition, particularly for prepubertal children and those in the early stages of sexual development. Use of DXA BMD would be reasonable if adjustments for body size, pubertal status, and skeletal maturity are made, but these additional assessments add significant complexity to the studies.

To date, the best method to evaluate bone architecture and content of the lumbar bones is CT. An additional advantage is the measure it gives also for the adjacent muscles. In this study, when the authors compared CT to DEXA measurements, they found that CT-BMC, which reflects the accurate bone mineral content, best correlates with DEXA-BMC and not with other measurements that take into account bone area or volume estimations of the lumbar bone in DEXA. They examined healthy children from 6 to 16 years of age with average height and weight. It is likely that the strength of the associations differs in other populations, as in children who are short, sick, overweight, etc. Since our 
major concern as pediatric endocrinologists is the 'true bone composition' in chronically ill patients and those who receive steroids, further studies in the populations are needed to define the best method for estimating bone health and composition in this subgroup and guide therapeutic interventions.

\title{
Constitutional delay of growth and puberty and bone quality
}

Peak bone mineral density is achieved several years after the completion of puberty. Puberty has an important role in the cumulative effect of bone mineral density. In the next studies, the questions of whether late puberty influences bone composition and content are addressed.

\section{The skeletal phenotype of men with previous constitutional delay of puberty}

\author{
Yap F, Hogler W, Briody J, Moore B, Howman-Giles R, Cowell CT \\ Institute of Endocrinology and Diabetes, The Children's Hospital at Westmead, Sydney, Australia \\ J Clin Endocrinol Metab 2004;89:4306-4311
}

Background: The aim of the study was to explore whether men with a history of constitutional delay (CD) of puberty are osteopenic.

Methods: 32 men with previous CD with 45 controls were evaluated. Auxology, bone mass, size, areal bone mineral density (aBMD) and volumetric bone mineral density at the lumbar spine (LS) and femoral neck (FN), and total-body and body segment measurements were determined by DEXA. The effects of prior androgen treatment were studied.

Results: Men with previous CD were shorter and had shorter height-adjusted arms compared with controls. Height-adjusted total-body bone mineral content, aBMD, and bone area were lower in CD men compared with controls. Segment length-adjusted BMC and bone area of arms and legs (but not trunk) were lower in CD men than in controls. However, they had normal LS and FN volumetric bone mineral density. Size-adjusted LS width and the hip cross-sectional area were lower than in controls. There was no difference in anthropometric or DEXA results between untreated and androgen-treated CD men.

Conclusion: Men with previous CD have normal LS and FN volumetric density but reduced total-body bone mass, which was explained by reduced limb bone mass and size. Together with the reduced LS bone width and hip cross-sectional area, these skeletal characteristics suggest impaired periosteal expansion during puberty. The skeletal phenotype of CD males may be altered by their late onset of puberty.

This work emphasizes the importance of analyzing various parameters for measuring bone density to have a full assessment of bone health. However it is important to keep in mind that the relationship of most of these parameters with fracture risk, as a gold standard of bone health, has not been established. In continuation with the previous paper, this article shows that in men with delayed puberty, volumetric bone mineral density in lumbar spine and femoral neck was normal. However, these men had lower areal bone mineral density (aBMD). The low aBMD may result from impaired periosteal expansion during puberty in this cohort. The lack of difference in anthropometric or DEXA results between untreated and androgen-treated constitutional delay men raises the question of what is the cause of impaired periosteal expansion. The next article addresses this question. 


\section{Estrogens are essential for male pubertal periosteal bone expansion}

Bouillon R, Bex M, Vanderschueren D, Boonen S

Division of Endocrinology, Katholieke Universiteit Leuven, Leuven, Belgium

roger.bouillon@med.kuleuven.ac.be

J Clin Endocrinol Metab 2004;89:6025-6029

Background: In order to explore the role of estrogen on bone maturation and composition, the skeletal response to estrogen therapy was studied in a 17-year-old boy with congenital aromatase deficiency.

Methods: DEXA assessed the areal bone mineral density (BMD) of the lumbar spine and femoral neck. Peripheral quantitative computed tomography at the ultradistal radius was measured before and after estrogen therapy.

Results: Estrogen therapy (1 mg estradiol valerate/day from age 17 until 20 years) normalized total and free testosterone and reduced the rate of bone remodeling. DEXA-assessed areal BMD of the LS and FN increased significantly (by $2 \%$ and $14 \%$, respectively), but pQCT at the ultradistal radius revealed no gain of either trabecular or cortical volumetric BMD. The increase in areal BMD was thus driven by an increase in bone size. Indeed, longitudinal bone growth (height, $+8.5 \%$ ) and especially cross-sectional area of the radius $(+46 \%)$ and cortical thickness $(+12 \%)$, increased markedly during estrogen treatment.

Conclusion: These findings demonstrate that androgens alone are insufficient, whereas estrogens are essential for the process of pubertal periosteal bone expansion typically associated with the male bone phenotype.

The observation of osteoporosis in aromatase deficiency is not new. However, this case study of aromatase deficiency allows us to learn about the significance of estrogen in bone maturity and architecture. Despite full pubertal development and supraphysiological levels of total and free testosterone, $x$-rays of the left wrist and hand revealed open epiphyses and a bone age of only 12 years. At the age of 17 years, administration of estrogen increased serum estradiol and normalized total and free testosterone, FSH, and LH. Serum osteocalcin was significantly reduced as well. During 3 years of treatment, body height increased by $8.5 \%$, from 176 to $191 \mathrm{~cm}$, and bone age matured from $125 / 12$ to $16 \% / 12$ years, with near closure of the epiphyses only observed at $205 / 12$ years of age. Although direct measurements of volumetric BMD using PQCT at the radius confirmed that volumetric density was largely unaffected by estrogen treatment at cortical sites such as radius and femoral neck, DEXA-assessed total area increased by 18 and $21 \%$, respectively, without a significant change in BMAD. This case is particularly interesting in the context of the current trend for clinical trials of aromatase inhibitors in pediatric endocrinology. A deficiency in aromatase can cause a delay in the closure of the epiphyses and a change in the bone architecture.

From these articles we can learn that individuals with delayed puberty have normal bone density, but impaired periosteal expansion. Estrogen therapy in subjects with estrogen deficiency, as examined by Bouillon et al., demonstrates the role of estrogens in periosteal expansion. It is therefore possible that low estrogen levels in children with delayed puberty may contribute to structural bone changes. A mini-review published in 2004 by Gennari et al. [6] discusses the role of aromatase activity in bone homeostasis in men. 


\section{Age at first oral contraceptive use as a major determinant of vertebral bone mass in female endurance athletes}

Hartard M, Kleinmond C, Kirchbichler A, Jeschke D, Wiseman M, Weissenbacher ER, Felsenberg D, Erben RG

Working Group MusculoSkeletal Interactions, Faculty of Sport Science, Technische Universität München,

Munich, Germany

Manfred.Hartard@t-online.de

Bone 35:4:863-841

Background: The aim of this retrospective analysis to examine the influence of low-dose monophasic oral contraceptives (OCs) on bone mineral density (BMD) of the femoral neck and of the spine in young female endurance athletes.

Methods: 75 regularly exercising endurance athletes aged 18-35 years (26.5 \pm 4.8 years) were included in this analysis. Data on training intensity, dietary intake, menarche, menstrual cycle disorders, years of $\mathrm{OC}$ use, and age at first OC use were determined by a self-report questionnaire. Clinical assessment included measurement of weight, height, spine, and hip BMD by dual-energy x-ray absorptiometry, and collection of a blood sample. The data from the study group was divided into the OC group when they reported OC use for $>3$ years in women $<22$ years of age, or when they reported OC use for $>50 \%$ of the time after menarche in women aged 22-35 years.

Results: No differences in age, weight, height, body mass index (BMI), body fat, menarche, training intensity, age at start of training, or any serum parameters between OC users and control subjects. However, OC users had 7.9\% lower spine BMD and 8.8\% lower proximal femur BMD $(\mathrm{p}<0.01$ for both sites). A stepwise model of multiple regression analysis revealed that the age at first OC use was found to be the best predictor of vertebral BMD, while the only significant predictor of femoral neck BMD was BMI.

Conclusion: OC use is associated with decreased BMD of the spine and the femoral neck in female endurance athletes. The early age at initiation of OC use may be an important risk factor for low peak bone mass in young women.

The authors show that using oral contraceptives may decrease bone mineral density of the spine and femoral neck. These results differ from a study previously conducted by Prior et al. [7], who did not find any effect of $O C$ on bone mineral density. The discrepancy may be due to the fact that the female subjects in this study were younger.

An attempt to explain why estrogen administration during the ages when peak bone mass is achieved may result in decreased bone mass, arises from the fact that estrogen treatment can suppress the ovarian function and by that reduce the androgen synthesis in the ovaries. Indirect evidence for androgen's role in female bone can be found from the high BMD in females with PCOS and from animal studies. In these studies, ovariectomy induced cancellous bone loss in estrogen-receptor knockout mice. This bone loss was prevented by dihydrotestosterone supplementation.

\section{Glucocorticoids and bone}

\section{Long-term, high-dose glucocorticoids and bone mineral content in childhood glucocorticoid-sensitive nephrotic syndrome}

Leonard MB, Feldman HI, Shults J, Zemel BS, Foster BJ, Stallings VA

Department of Pediatrics, Children's Hospital of Philadelphia, Philadelphia, Pa., USA

leonard@email.chop.edu

N Engl J Med 2004;351:868-875

Background: The aim of the study was to determine the effects of long-term treatment with glucocorticoids on bone mineral content in children with glucocorticoid-sensitive nephrotic syndrome. 
Methods: Dual-energy x-ray absorptiometry (DEXA) of the whole body and spine was performed in 60 children and adolescents with the nephrotic syndrome and 195 control subjects.

Results: Patients receiving intermittent treatment with high-dose glucocorticoids were shorter and had a greater body mass index than controls. The bone mineral content of the spine, adjusted for bone area, age, sex, degree of maturation (Tanner stage), and race, did not differ significantly between patients and controls. However, after adjustment for the z-score for body-mass index, the bone mineral content of the spine was significantly lower in patients than in controls. Whole-body bone mineral content was significantly higher in patients than in controls.

Conclusions: Intermittent treatment with high-dose glucocorticoids during growth does not appear to be associated with severe deficits in the bone mineral content of the spine or whole body. Glucocorticoidinduced increases in body mass index were associated with increased whole-body bone mineral content and maintenance of the bone mineral content of the spine.

In adults, glucocorticoids cause rapid, dose-dependent bone loss and an increased risk of fracture. In this study, Leonard and colleagues report that intermittent glucocorticoid treatment even in high dosages in glucocorticoid-sensitive nephrotic syndrome was not associated with significant reduction in bone mineral content at the lumbar spine or whole body. The patients had significantly lower z-scores for height and significantly higher z-scores for weight and BMI. Apparently, whole-body $B M C$ was significantly greater in the patients than in the controls. However, when the spine BMC was adjusted to BMI, the results demonstrated that the patients had significantly lower BMC in the spine as compared with the controls. Separating BMC into cortical and trabecular bones demonstrated that the trabecular BMC of the spine was decreased while the cortical BMC was higher than that in controls, after adjustment for the BMI. This finding is consistent with reports that cortical BMC increases with mechanical loading in children, and on the other hand, that trabecular bone is more susceptible than cortical bone to the effects of glucocorticoids.

It should be emphasized that although intermittent treatment with high-dose glucocorticoids in children was not associated with deficits in whole-body BMC in this study, changes in the bone 'micro architecture' of the spine were detected.

Although the results in this cohort of patients show a minor reduction in bone mineral density in the trabecular bone of the spine, we should remember that patients who are treated with high doses of glucocorticoids for long periods present with devastating reduction in bone mass, especially in the spine, that increases the risk of spinal compression fractures.

\section{Calcium and bone}

The Food and Nutrition Board of the US National Academy of Sciences recommends that adequate dietary intake of calcium is necessary in children and adolescents for the development of peak bone mass, prevention of fractures and osteoporosis later in life. The current recommended intake for children 9-18 years of age is $1,300 \mathrm{mg} /$ day [8]. At higher intake, additional calcium is mostly excreted. Peak calcium accretion is attained at the ages of 12.5 years in girls and 14 years in boys and for this age group the National Academy of Sciences has set an upper limit of 2,500 mg/day [9]. Despite this, the percent of children taking adequate calcium in the USA declines dramatically after the second year of life, reaching its nadir between 12 and 19 years of age, when documented intakes in this age group approximate only $700-1,000 \mathrm{mg} /$ day. In fact, only $10 \%$ of adolescent girls achieve the recommended adequate dietary intake of calcium. In the next three studies, the effect of calcium supplementation was examined in prepubertal and pubertal boys and in prepubertal girls. 


\title{
Calcium supplementation increases stature and bone mineral mass of 16- to 18-year-old boys
}

Prentice A, Ginty F, Stear SJ, Jones SC, Laskey MA, Cole TJ

Medical Research Council Human Nutrition Research, Elsie Widdowson Laboratory, Cambridge, and Centre for Paediatric Epidemiology and Biostatistics, Institute of Child Health, London, UK ann.prentice@mrc-hnr.cam.ac.uk

J Clin Endocrinol Metab 2005;90:3153-3161

Background: The effect of calcium carbonate supplementation on bone growth and mineral accretion was studied in pubertal boys.

Methods: 143 boys aged 16-18 years were randomized to $1,000 \mathrm{mg} \mathrm{Ca} /$ day or a matching placebo for 13 months. Anthropometry and DEXA of the whole body, lumbar spine, hip, and forearm were performed before, during, and after the intervention.

Results: The intervention resulted in greater bone mineral content $(\mathrm{BMC})$ of the whole body $(+1.3 \%$, $\mathrm{p}=0.02)$, lumbar spine $(+2.5 \%, \mathrm{p}=0.004)$, and hip (total $+2.3 \%, \mathrm{p}=0.01$; neck $+2.4 \%, \mathrm{p}=0.02$; intertrochanter $+2.7 \%, \mathrm{p}=0.01)$. This was associated with greater height $(+0.4 \%, \mathrm{p}=0.0004$, equivalent to $7 \mathrm{~mm})$, lean mass $(+1.3 \%, \mathrm{p}=0.02)$, and lumbar spine bone area $(+1.5 \%, \mathrm{p}=0.003)$. The increases in BMC diminished after size adjustment, suggesting that the intervention effect was mediated through an effect on growth. The BMC response at the intertrochanter was greater in subjects with high physical activity $(+4.4 \%, \mathrm{p}=0.05)$. There were no other significant interactions with physical activity, plasma testosterone, calcium intake, or tablet compliance.

Conclusion: Calcium carbonate supplementation of adolescent boys increased skeletal growth, resulting in greater stature and bone mineral acquisition.

In prepubertal boys, supplementation of $850 \mathrm{mg}$ calcium per day in addition to the normal diet resulted in the consumption of $1.5 \mathrm{~g}$ of calcium per day. One year of such a calcium supplementation caused an increase in bone-mineral density in several appendicular skeletal sites by the end of the study year. However, the lumbar bone density did not change. This increase in bone-mineral density is not lost after 1 year following the end of intervention [10]; the question remains whether this benefit will be maintained until attainment of peak bone mass. In this study, as compared with a previous one by the same group, it was observed that calcium supplementation in pubertal boys between the ages of 16-18 resulted in acceleration of bone growth and higher stature. Although the difference was small, it was statistically significant. BMC also increased. However, adjustment of BMC to bone size did not reveal any difference in the BMD. These results are different from those observed in the prepubertal age, when calcium supplementation resulted in an increased aBMD in appendicular skeletal sites but not in the lumbar spine. As expected, physical activity in this age group caused a significant increase in inter-trochlear bone mineral content. The observations in this study raise a question regarding the anabolic mechanism of calcium supplement, expressed in an increase in height, lean body mass and body size. We do not know what the long-term consequences of higher BMC and bigger bones in relation to bone strength and the occurrence of future bone fractures are.

\section{Interaction between calcium intake and menarcheal age on bone mass gain: an 8-year follow-up study from prepuberty to postmenarche}

\author{
Chevalley T, Rizzoli R, Hans D, Ferrari S, Bonjour JP \\ Service of Bone Diseases (WHO Collaborating Center for Osteoporosis Prevention), \\ Department of Rehabilitation and Geriatrics, Geneva, Switzerland \\ Thierry.Chevalley@hcuge.ch \\ J Clin Endocrinol Metab 2005;90:44-51
}

Background: Both late menarcheal age and low calcium intake during growth are risk factors for osteoporosis, probably by impairing peak bone mass. In this study it was investigated whether lasting gain in areal bone mineral density $(\mathrm{aBMD})$ in response to increased $\mathrm{Ca}$ intake varies according to menarcheal age and, conversely, whether $\mathrm{Ca}$ intake could influence menarcheal age.

Methods: In an initial study, 144 prepubertal girls were randomized in a double-blind controlled trial to receive either a $\mathrm{Ca}$ supplement $(\mathrm{Ca}-\mathrm{suppl})$ of $850 \mathrm{mg} /$ day or placebo from age 7.9-8.9 years. Mean 
aBMD was determined by DEXA at six sites (radius metaphysis, radius diaphysis, femoral neck, trochanter, femoral diaphysis, and L2-L4). After 12 months, aBMD of the prepubertal girls was significantly greater in the Ca-suppl group than in the placebo group, $27 \mathrm{vs.} 21 \mathrm{mg} / \mathrm{cm}^{2}$. In 122 girls followed up, menarcheal age was recorded, and aBMD was determined at 16.4 years of age.

Results: Menarcheal age was lower in the Ca-suppl than in the placebo group (5.4 months; $\mathrm{p}=0.048$ ). Menarcheal age was negatively correlated to $\mathrm{Ca}$ intake $(\mathrm{r}=-0.35 ; \mathrm{p}<0.001)$ and to aBMD gains from age 7.9-16.4 years at all skeletal sites. Mean aBMD was significantly $(p=0.004)$ greater in the Ca-suppl than in the placebo group $\left(27\right.$ vs. $\left.21 \mathrm{mg} / \mathrm{cm}^{2}\right)$. The positive effect of Ca-suppl on the mean aBMD gain from baseline remained significantly greater in girls with early menarcheal age below 13 years $\left(12.1 \pm 0.5\right.$ years): placebo $286 \pm 36 \mathrm{mg} / \mathrm{cm}^{2}$; Ca-suppl $317 \pm 46(\mathrm{p}=0.009)$, but not in those with late age, $>13.0$ years (late menarcheal age (13.9 \pm 0.5 years): placebo $284 \pm 58$; Ca-suppl $276 \pm 50(\mathrm{p}>0.05)$.

Conclusions: The level of $\mathrm{Ca}$ intake during prepuberty may influence the timing of menarche, which, in turn, could influence long-term bone mass gain in response to Ca supplementation. Thus, both determinants of early menarcheal age and high $\mathrm{Ca}$ intake may positively interact on bone mineral mass accrual.

Providing girls with calcium supplementation of $850 \mathrm{mg} /$ day for 1 year just before entering puberty (between 7.9 and 8.9 years of age) caused an increase in aBMD throughout the skeleton, including the spine, at the end of treatment. Surprisingly, the age of menarche was lower in the supplemented group (by 5.4 months). The main effect of calcium supplements on aBMD was observed in girls who experienced an earlier onset of menarche, below the age of 13 .

The question that evolves is what are the connections between calcium supplementation and the early menarche? Is it the anabolic effects of the calcium supplements, as discussed in the previous articles? Is calcium supplementation good or bad for the girls? According to aBMD results, it is good. However, we should also keep in mind a new report that girls with early menarche exhibit hypertension and glucose intolerance as compared with those having later maturity, independently of body composition [11].

\section{New genes and mechanisms Phosphorus homeostasis and bone}

The two main regulators in phosphorus homeostasis are PTH and the osteoblast-derived hormone FGF23. The clinical conditions resulting from hyper- or hypoparathyroidism are relatively frequent and were studied extensively. On the other hand, the conditions related to excessive or deficient FGF23 activity are relatively new (table 1); they were recently explored and continue to be studied. There are several disease conditions that are associated with increased FGF23 activity. Tumor-induced osteomalacia (TIO) is a paraneoplastic syndrome of renal phosphate wasting characterized by low phosphorus levels due to reduced renal reabsorption, inappropriately low calcitriol levels, with normal calcium and PTH levels. This clinical syndrome is due to overexpression of FGF23 in the TIO tumor. Similar to TIO, patients with extensive fibrous dysplasia exhibit elevated serum FGF23 levels. Another two inherited renal phosphate-wasting syndromes, due to enhanced FGF23 activity, are autosomal dominant hypophosphatemic rickets (ADHR) and X-linked hypophosphatemic rickets (XLHR). ADHR results from a missense mutation in the FGF23 gene that prevents its degradation by the metalloprotease Phex, whereas XLHR is due to a mutation in the PHEX gene.

A clinical metabolic syndrome that is manifested as a mirror image to TIO, ADHR, and XLHR, as reported in this Yearbook, is familial tumoral calcinosis (FTC). This syndrome is characterized by ectopic calcification, hyperphosphatemia due to enhanced renal phosphate retention, and inappropriately normal or elevated calcitriol. Two new mechanisms that are related to this syndrome were discovered in the last year. Topaz et al. identified that FTC is due to a loss of function mutation in the GALNT3 gene and this observation was followed by description of a new mutation by lchikawa et al. Another mechanism reported by Larson et al. is due to a loss-of-function mutation in the FGF23 gene that results with a truncated FGF23 protein. 
Table 1. FGF23: (A) overproduction and gain of function and (B) underproduction and loss of function

A

\begin{tabular}{llll}
\hline TIO & FD & ADHR & XLHR \\
\hline $\begin{array}{c}\text { Tumor-induced } \\
\text { Osteomalacia }\end{array}$ & Fibrous dysplasia & $\begin{array}{l}\text { Autosomal dominant } \\
\text { hypophosphatemic } \\
\text { rickets }\end{array}$ & $\begin{array}{l}\text { X-linked hypophosphatemic } \\
\text { rickets }\end{array}$ \\
$\begin{array}{c}\text { Overexpression } \\
\text { of FGF23 in the } \\
\text { tumor }\end{array}$ & $\begin{array}{l}\text { Overexpression } \\
\text { of FGF23 in the } \\
\text { tissue }\end{array}$ & $\begin{array}{l}\text { Missense mutation in the } \\
\text { FGF23 gene that prevents } \\
\text { its degradation by the } \\
\text { metalloprotease Phex }\end{array}$ & Mutation in the PHEX gene \\
& &
\end{tabular}

Renal phosphate wasting characterized by low phosphorus levels due to reduced renal reabsorption, inappropriately low calcitriol levels, with normal calcium and PTH levels

\section{$B$}

\begin{tabular}{lll}
\hline FTC & FTC & HHS \\
\hline Familial tumoral calcinosis & $\begin{array}{c}\text { Familial tumoral } \\
\text { calcinosis } \\
\text { Loss of function mutation } \\
\text { in the GALNT3 gene }\end{array}$ & $\begin{array}{c}\text { Hyperphosphatemia- } \\
\text { hyperostosis syndrome } \\
\text { Homozygous splice site } \\
\text { mutation in GALNT3 }\end{array}$ \\
$\begin{array}{l}\text { FGF23 gene, truncated } \\
\text { protein }\end{array}$ & $\begin{array}{l}\text { Elevated serum phosphate } \\
\text { Ectopic calcification, hyperphosphatemia due to enhanced renal } \\
\text { phosphate retention, and inappropriately normal or elevated } \\
\text { calcitriol }\end{array}$ & $\begin{array}{l}\text { levels and repeated } \\
\text { attacks of acute, painful } \\
\text { swellings of the long } \\
\text { bones with radiological } \\
\text { evidence of periosteal } \\
\text { reaction and cortical } \\
\text { hyperostosis }\end{array}$ \\
& \\
&
\end{tabular}

\section{Mutations in GALNT3, encoding a protein involved in O-linked glycosylation, cause familial tumoral calcinosis}

Topaz O, Shurman DL, Bergman R, Indelman M, Ratajczak P, Mizrachi M, Khamaysi Z, Behar D, Petronius D, Friedman V, Zelikovic I, Raimer S, Metzker A, Richard G, Sprecher E

Department of Dermatology, Rambam Medical Center, Haifa, Israel e_sprecher@rambam.health.gov.il

Nat Genet 2004;36:579-581

Background: Familial tumoral calcinosis (FTC) is a severe autosomal recessive metabolic disorder that manifests with hyperphosphatemia and massive calcium deposits in the skin and subcutaneous tissues. Methods: Twelve individuals with FTC from two large kindreds of Druze and African-American origin were studied. Using linkage analysis, the gene underlying FTC was mapped to $2 \mathrm{q} 24-\mathrm{q} 31$. This region includes the gene GALNT3, which encodes a glycosyltransferase responsible for initiating mucin-type O-glycosylation.

Results: Sequence analysis of GALNT3 identified biallelic deleterious mutations in all individuals with FTC. Conclusions: Defective post-translational modification of FGF23 underlies the disease.

Polypeptide N-acetylgalactosaminyltransferase (GALNT3) is one of several enzymes that catalyze the

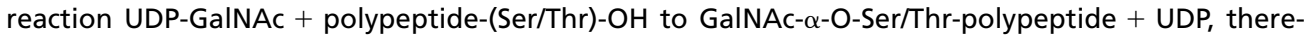
by initiating O-glycosylation of serine and threonine residues on an array of glycoproteins. This enzyme is important in the post-translational modification of FGF23 for its proper function. 


\title{
A novel recessive mutation in fibroblast growth factor-23 causes familial tumoral calcinosis
}

\author{
Larsson T, Yu X, Davis SI, Draman MS, Mooney SD, Cullen MJ, White KE \\ Department of Medical and Molecular Genetics, Indiana University School of Medicine, Indianapolis, Ind., USA \\ kenewhit@iupui.edu.
}

J Clin Endocrinol Metab 2005;90:2424-2427

Background: Tumoral calcinosis (TC) patients are often hyperphosphatemic with inappropriately normal or elevated serum calcitriol levels and have ectopic and vascular calcifications, a phenotype similar to that of FGF23 null mice. Therefore, the goal of the present studies was to test whether FGF23 was a candidate gene for TC.

Methods: Two sisters in a consanguineous TC family had hyperphosphatemia and normal calcitriol levels with characteristic ectopic and vascular calcifications. Surprisingly, these patients had low-normal intact serum FGF23 levels but demonstrated FGF23 concentrations approximately 40 times normal when assessed with a C-terminal FGF23 serum assay.

Results: Mutational analyses identified a homozygous S71G mutation in FGF23 in the TC patients, which was not found in control alleles. Modeling demonstrated that the S71G mutation most likely destabilizes full-length FGF23.

Conclusions: FGF23 mutations can lead to TC. FGF23 may adopt an unstable conformation in some TC patients, possibly leading to non-functional FGF23 protein.

FGF23 is now implicated in disorders of both phosphorus deficiency and excess. However, the role of FGF23 in daily phosphorus homeostasis is not fully clarified. The next study attempted to address this issue.

\section{Fibroblast growth factor-23 relationship to dietary phosphate and renal phosphate handling in healthy young men}

Ferrari SL, Bonjour JP, Rizzoli R

Service of Bone Diseases, Geneva University Hospital, Geneva, Switzerland

serge.ferrari@medecine.unige.ch

J Clin Endocrinol Metab 2005;90:1519-1524

Background: PTH and FGF23 are the main factors that control renal handling of inorganic phosphate (Pi). The question of whether phosphate intake has any effect on FGF23 activity is not fully understood. Methods: Twenty-nine healthy males were subjected to a 5-day low-phosphate diet and a phosphate binder, followed by a high-phosphate diet including supplements. Concomitant modifications in calcium intake allowed minimizing PTH changes in response to dietary phosphate.

Results: Serum FGF23 levels significantly decreased on the low-phosphate diet, then increased with the oral phosphate load. Changes in FGF23 were positively correlated with changes in 24-hour urinary Pi excretion and negatively correlated with changes in the maximal tubular reabsorption of $\mathrm{Pi}$ and calcitriol, whereas PTH was not. FGF23 was positively correlated to serum osteocalcin.

Conclusions: FGF23 may be implicated in the physiological regulation of Pi homeostasis in response to dietary phosphate changes, independent of PTH.

The authors show that an oral phosphate load increases circulating FGF23 levels. These changes in serum FGF23 correlate positively with osteocalcin levels and inversely correlate with the tubular reabsorption of $\mathrm{Pi}$ and plasma calcitriol levels, suggesting that the bone is involved in the regulation of phosphate homeostasis. It looks like the bone has a 'phophorus-stat' that plays a role in the homeostasis of phosphorus in a very tight margin. Phosphorus has a major role in endochondral ossification and in bone homeostasis. Pi induces apoptosis of the terminal hypertrophic chondrocyte in a dose- and time-dependent manner. Low phosphate is the major determinant of decreased chondrocyte apoptosis that leads to rachitic changes in the growth plate. In the osteoblast, the phosphate signal ties osteopontin expression to alkaline phosphatase. Free phosphate enters the cell through a $\mathrm{Na}$-dependent phosphate transporter, which results in the up-regulation of osteopontin RNA levels. Osteopontin modulates hydroxyapatite crystal elongation during bone formation. The tight link 
between osteopontin expression and phosphate levels is combined with the ability of osteopontin to modulate calcium levels.

\section{A new member in the phosphtonin family}

\section{Fibroblast growth factor 7: an inhibitor of phosphate transport derived from oncogenic osteomalacia-causing tumors}

Carpenter TO, Ellis BK, Insogna KL, Philbrick WM, Sterpka J, Shimkets R

Endocrine Sections of the Department of Pediatrics, Yale University School of Medicine, New Haven, Conn., USA

thomas.carpenter@yale.edu

J Clin Endocrinol Metab 2005;90:1012-1020

Background: Oncogenic osteomalacia (OO), a tumor-associated phosphate-wasting syndrome, provides an opportunity to identify regulators of renal phosphate homeostasis.

Methods: By comparing RNA from tumor-derived cultures expressing inhibitory activity with RNA from tumor-derived cultures in which inhibitory activity was not evident, the authors identified candidate mRNAs specifically expressed by cultures inhibiting renal phosphate transport.

Results: Fibroblast growth factor 7 (FGF7) was a potent and direct inhibitor of phosphate uptake in vitro. A neutralizing monoclonal antibody to FGF7 reversed FGF7-dependent phosphate transport inhibition and inhibitory activity. Only small amounts of FGF23 were present in inhibitory conditioned medium, comparable to concentrations found in conditioned medium with no phosphate transport inhibitory activity.

Conclusions: Members of the FGF family (other than FGF23) are expressed by OO-associated tumors and may play a role in mediating this syndrome.

In this study the authors show in an elegant in-vitro study that tumors associated with phosphate wasting secrete not only FGF23 but also other members of the FGF family, such as FGF7. FGF7 is a 28-kDa member of the FGF family, and has been demonstrated to be an active stimulus in wound healing and highly expressed in keratinocytes. FGF7 is expressed in other epithelial tissues, including gastrointestinal epithelium, transitional urothelial cells, and type II pneumocytes. Its expression is increased in the setting of inflammatory bowel disease. FGF7 and FGF23 act through different receptors. FGF7 is believed to selectively activate the FGFR2b receptor, whereas FGF23 has been shown to bind to FGFR2C and FGFR3c. It appears that activation of different FGF receptors is able to mediate the inhibitory effects of FGFs on Pi transport in renal epithelium. We may finally understand several enigmatic conditions that are associated with hypophosphatemia. The rule remains that when you encounter any electrolyte abnormality, such as hypophosphatemia, you check urinary excretion first.

\section{Vitamin D - new genes}

\section{Genetic evidence that the human CYP2R1 enzyme is a key vitamin D 25-hydroxylase}

Cheng JB, Levine MA, Bell NH, Mangelsdorf DJ, Russell DW

Department of Molecular Genetics, University of Texas Southwestern Medical Center, Dallas, Tex., USA

Proc Natl Acad Sci USA 2004; 101:7711-7715

Background: The identity of the hepatic 25-hydroxylase has remained unclear for $>30$ years. The microsomal CYP2R1 protein as a potential candidate for the liver vitamin D 25-hydroxylase based on the enzyme's biochemical properties, conservation, and expression pattern was previously identified. 
Methods: A patient with low circulating levels of 25-hydroxyvitamin D and classic symptoms of vitamin D deficiency was studied.

Results: This individual was found to be homozygous for a transition mutation in exon 2 of the CYP2R1 gene on chromosome $11 \mathrm{p} 15.2$. The inherited mutation caused the substitution of a proline for an evolutionarily conserved leucine at amino acid 99 in the CYP2R1 protein and eliminated vitamin D 25hydroxylase enzyme activity.

Conclusion: These data identify CYP2R1 as a biologically relevant vitamin D 25-hydroxylase and reveal the molecular basis of a human genetic disease, selective 25 -hydroxyvitamin D deficiency.

The enzymatic steps in the vitamin $D_{3}$ biosynthetic pathway have been known for 30 years, but the enzyme catalyzing the 25-hydroxylation step in the liver had not been identified. CYP27A1 and CYP2R1 were candidates for the vitamin D 25-hydroxylase. Both enzymes are expressed in the liver and are conserved among species known to have an active vitamin D signaling pathway. However, mutations in the human and mouse genes encoding the mitochondrial CYP27A1 protein impair bile acid synthesis and results in a cholesterol storage disease, cerebrotendinous xanthomas, with normal vitamin D metabolism. In this study, the missing part in the picture of vitamin D metabolism was found; the authors describe the molecular analysis of a patient with abnormally low plasma levels of $25-\mathrm{OHD}_{3}$ and classic symptoms of vitamin $\mathrm{D}$ deficiency. This individual is homozygous for a substitution mutation in exon 2 of CYP2R1 gene, which encodes for the microsomal enzyme that was identified in 2003.

\title{
New concept - Vitamin D receptor action
}

\section{Ligand-independent actions of the vitamin D receptor maintain hair follicle homeostasis}

\author{
Skorija K, Cox M, Sisk JM, Dowd DR, MacDonald PN, Thompson CC, Demay MB \\ Endocrine Unit, Massachusetts General Hospital and Harvard Medical School, Boston, Mass., USA \\ Mol Endocrinol 2005; 19:855-862
}

Background: Alopecia is a feature of vitamin D receptor (VDR) mutations in humans and in VDR null mice. Methods: To determine which functional domains of the VDR are required for hair cycling, mutant VDR transgenes were targeted to the keratinocytes of VDR null mice.

Results: Keratinocyte-specific expression of a VDR transgene with a mutation in the hormone-binding domain that abolishes ligand binding restores normal hair cycling in VDR null mice, whereas a VDR transgene with a mutation in the activation function-2 domain that impairs nuclear receptor coactivator recruitment results in a partial rescue. Mutations in the nuclear receptor corepressor Hairless are also associated with alopecia in humans and mice. Hairless binds the VDR, resulting in transcriptional repression. Neither VDR mutation affects Hairless interactions or its ability to repress transcription. Conclusion: These studies demonstrate that the effects of the VDR on the hair follicle are ligand independent and point to novel molecular and cellular actions of this nuclear receptor.

This elegant study expands our understanding regarding vitamin $D$ receptor action. The presence of alopecia in humans and mice with mutant VDR suggests that the VDR has a major role in hair follicle homeostasis. The fact that humans and mice with $1 \alpha$-hydroxylase deficiency and with no detectable circulating levels of calcitriol do not develop alopecia indicates that this homeostasis in the hair follicle is not ligand-dependent on the effects of the VDR. Interestingly, a mutation of the Hairless gene in mice and humans results in alopecia similar to that seen in the VDR null mice. Recent studies have demonstrated that the Hairless protein interacts with VDR and dramatically represses both basal and ligand-dependent VDR-mediated transactivation in transient gene expression assays. Furthermore, Hairless is expressed in the same hair follicle cells as the VDR, suggesting that the physical and functional interaction of Hairless and VDR occurs in vivo. This study demonstrates that VDR mutants that maintain the DNA binding domain retain the ability to interact with Hairless and this indicates that the Hairless-VDR complex may play a critical role in the maintenance of the hair cycle. 
This work shows that VDR harbors coactivator and corepressor activities that are ligand dependent and non-ligand dependent. The DNA binding domain mediates both activities.

Ligand binding by the VDR promotes its heterodimerization with RXR and binding to target DNA sequences via the DNA-binding domain, that will end in recruitment of nuclear receptor coactivators, via the activation function-2 (AF2) domain. This leads to chromatin remodeling and interactions with the basal transcriptional apparatus, resulting in the induction of gene transcription. On the other hand, this study demonstrated that the VDR DNA binding domain, regardless of ligand occupancy, interacts with Hairless, a protein that functions as a corepressor and has a role in the hair follicle homeostasis.

\section{Review - Cystic fibrosis and bone health}

\section{Guide to bone health and disease in cystic fibrosis}

Aris RM, Merkel PA, Bachrach LK, Borowitz DS, Boyle MP, Elkin SL, Guise TA, Hardin DS, Haworth CS, Holick MF, Joseph PM, O'Brien K, Tullis E, Watts NB, White TB

CB 7020, 4131 Bioinformatics, University of North Carolina, Chapel Hill, N.C., USA aris@med.unc.edu

J Clin Endocrinol Metab 2005;90:1888-1896

Cystic fibrosis (CF) is the most common genetic disease within the Caucasian population and leads to premature respiratory failure. Bone disease has emerged as a common complication in long-term survivors of CF. Prevention and treatment of CF-related bone disease must address several issues as decreased absorption of fat-soluble vitamins due to pancreatic insufficiency, altered sex hormone production, chronic lung infection with increased levels of bone-active cytokines, physical inactivity, and glucocorticoid therapy for poor bone health. This review is a condensed and updated summary of the "Guide to Bone Health and Disease in Cystic Fibrosis: A Consensus Conference", a statement that evolved from a meeting convened by the Cystic Fibrosis Foundation in May 2002 to address the pathogenesis, diagnosis, and treatment of bone disease in CF.

In this consensus paper the authors have presented a comprehensive update concerning bone health and treatment in CF patients. The article encompasses great detail and addresses the clinical correlates of low bone mass, bone histomorphometry, bone accrual and loss and bone turnover in CF patients.

The pathogenesis of bone disease in CF patients is discussed within the following topics: pancreatic exocrine insufficiency and malabsorption, pancreatic endocrine insufficiency, physical inactivity, delayed puberty or early gonadal failure, chronic infection, glucocorticoids, lung transplantation and immunosuppressant therapy. The authors provide screening and treatment protocols for bone disease in CF. Those of us who see CF patients for endocrine consultations have a chance here for a detailed update. 


\section{Single-dose pharmacokinetics and tolerability of alendronate 35- and 70-mg tablets in children and adolescents with osteogenesis imperfecta type I}

Ward LM, Denker AE, Porras A, Shugarts S, Kline W, Travers R, Mao C, Rauch F, Maes A, Larson P, Deutsch P, Glorieux FH

Genetics Unit, Shriners Hospital for Children, and McGill University, Montreal, Que., Canada; Merck \& Co. Inc., Whitehouse Station, N.J., USA

J Clin Endocrinol Metab 2005;90:4051-4056

Background: The pharmacokinetics and single-dose tolerability of alendronate (ALN) was evaluated in children with mild osteogenesis imperfecta (OI).

Methods: Twenty-four children (aged 4-16 years, 8 girls) received intravenous ALN at a dose of $125 \mu \mathrm{g}$ as well as oral ALN in a two-period, randomized crossover study, with doses separated by a 2-week washout. Patients weighing $<40 \mathrm{~kg}$ received ALN $35 \mathrm{mg}$ orally while those weighing $\geq 40 \mathrm{~kg}$ received oral ALN $70 \mathrm{mg}$. Urine was collected for $24 \mathrm{~h}$ following drug administration to determine the total urinary excretion of ALN and oral bioavailability.

Results: The total urinary excretion of ALN following the intravenous dose was similar for both weight groups. The mean oral bioavailability $(95 \%$ confidence interval) was $0.43 \%(0.28 \%, 0.64 \%)$ for patients weighing $<40 \mathrm{~kg}$ and $0.56 \%(0.36 \%, 0.87 \%)$ for patients weighing $\geq 40 \mathrm{~kg}$. Eighteen patients reported clinical adverse experiences, most commonly headache, nausea, fever and abdominal pain. Marginal decreases in absolute lymphocyte count and serum alkaline phosphatase at post-study compared with baseline were observed.

Conclusion: The oral bioavailability of ALN 35- and 70-mg tablets was $<0.6 \%$, comparable to adult studies. The drug was generally well tolerated.

Ward et al. found that the bioavailability of alendronate (ALN) in children and adolescence with osteogenesis imperfecta type I is $<1 \%$. The bioavailability variation of oral ALN by a factor of $>10$ may result from individual variation or from dosages that were not weight adjusted. Overall, ALN was well tolerated and it is very tempting to treat patients with a one-weekly pill. Further studies are needed to assess the longer term tolerability, safety and efficacy of ALN in children with OI and osteoporosis through randomized, placebo-controlled studies.

\section{New observations in bone metabolism}

\section{Adiponectin and its receptors are expressed in bone-forming cells}

Berner HS, Lyngstadaas SP, Spahr A, Monjo M, Thommesen L, Drevon CA, Syversen U, Reseland JE Oral Research Laboratory, Institute for Clinical Dentistry, University of Oslo, Oslo, Norway

hberner@odont.uio.no

Bone 2004;35:842-849

Background: Adiponectin is mainly synthesized and secreted exclusively by the adipose tissue, and is reported to influence energy homeostasis and insulin sensitivity.

Methods: Adiponectin expression and its receptors were investigated in primary human osteoblasts from femur and tibia.

Results: Adiponectin transcription, translation, and secretion, as well as expression of its receptors, AdipoR1 and AdipoR2, were found in primary human osteoblasts from femur and tibia bone-forming cells. The phenotype of bone cells was confirmed by the high expression levels of alkaline phosphatase, collagen type 1, osteocalcin, and CD44, and the formation of mineralization nodules. Immunostaining with monoclonal antibodies also demonstrated the presence of adiponectin in human osteosarcoma cells and normal osteoblasts. Both mRNA expression and secretion of adiponectin to the medium increased during differentiation of human osteoblasts in culture. The adiponectin mRNA level increases 
in osteoblasts cultured in the presence of dietary fatty acids and supplementation of culture medium with recombinant adiponectin enhances the proliferation of murine osteoblasts.

Conclusions: These findings suggest a functional role in bone homeostasis.

In the Yearbook 2005, we discussed the common stem cells for adipocyte and osteocyte, and how differentiation into these two tissues is regulated. We now learn that both tissues express adiponectin. Adiponectin expression in osteoblasts is much lower than in subcutaneous adipose tissue, indicating that adipocytes are still the major contributor to circulating adiponectin. However, this study clearly demonstrates that adiponectin is not adipocyte-specific. Adiponectin may, by binding to its receptors, directly influence the metabolism of cells in bone. The regulation of bone metabolism by adipokines is largely unknown, and the observed expression and secretion of adiponectin by boneforming cells add more complexity, and redundancy, to this intriguing issue.

\section{Lactoferrin is a potent regulator of bone cell activity and increases bone formation in vivo}

Cornish J, Callon KE, Naot D, Palmano KP, Banovic T, Bava U, Watson M, Lin JM, Tong PC, Chen Q, Chan VA, Reid HE, Fazzalari N, Baker HM, Baker EN, Haggarty NW, Grey AB, Reid IR

Department of Medicine, University of Auckland, Auckland, New Zealand j.cornish@auckland.ac.nz

Endocrinology 2004;145:4366-4374

Background: Many physiological functions have been proposed for lactoferrin, including the regulation of intestinal iron uptake, antimicrobial activity, regulation of cellular growth and differentiation, antiinflammatory and immunomodulatory activities, and protection against cancer. The precise mechanism of action of lactoferrin in many of these processes remains poorly understood. The aim of this study was to assess the role of lactoferrin on bone metabolism.

Methods: The effect of lactoferrin on osteoblast and osteoclasts were studied in vitro as well as in vivo adult mice.

Results: Lactoferrin produced large, dose-related increases in thymidine incorporation in primary or cell line cultures of human or rat osteoblast-like cells, at physiological concentrations $(1-100 \mu \mathrm{g} / \mathrm{ml})$. Maximal stimulation was 5-fold above control. Lactoferrin also increased osteoblast differentiation and reduced osteoblast apoptosis by up to $50-70 \%$. Similarly, lactoferrin stimulated proliferation of primary chondrocytes. Purified, recombinant, human, or bovine lactoferrins had similar potencies. In mouse bone marrow cultures, osteoclastogenesis was dose-dependently decreased and was completely arrested by lactoferrin. In contrast, lactoferrin had no effect on bone resorption by isolated mature osteoclasts. Lactoferrin was administered over calvariae of adult mice for 5 days. New bone formation, assessed using fluorochrome labels, was increased 4-fold by a 4-mg dose of lactoferrin.

Conclusion: Lactoferrin has powerful anabolic, differentiating, and antiapoptotic effects on osteoblasts and it inhibits osteoclastogenesis. Lactoferrin is a potential therapeutic target in bone disorders such as osteoporosis and is possibly an important physiological regulator of bone growth.

Lactoferrin is an iron-binding glycoprotein present in epithelial secretions, such as milk, and in the secondary granules of neutrophils. This study provides the first evidence that lactoferrin is a promoter of osteoblast growth. In addition, it shows that lactoferrin is an inhibitor of osteoclastogenesis in vitro and increases local bone formation in vivo. Lactoferrin acts through low-density lipoprotein receptor-related protein-1, a member of the low-density lipoprotein-related receptor family. These receptors, which are expressed in primary cultures of osteoblasts as well as in osteoblastic cell lines, have a large number of potential ligands, so they represent a novel pathway by which many factors might impact bone cell function. Here is a link between iron metabolism, child growth and bone health. This raises also the possibility that lactoferrin might serve new therapeutic purposes.

References

1. Kroger H, Vainio P, Nieminen J, Kotaniemi A: Comparison of different models for interpreting bone mineral density measurements using DXA and MRI technology. Bone 1995;17:157-159. 
2. Prentice A, Parsons TJ, Cole TJ: Uncritical use of bone mineral density in absorptiometry may lead to size-related artifacts in the identification of bone mineral determinants. Am J Clin Nutr 1994;60:837-842.

3. Warner JT, Cowan FJ, Dunstan FD, Evans WD, Webb DK, Gregory JW: Measured and predicted bone mineral content in healthy boys and girls aged 6-18 years: adjustment for body size and puberty. Acta Paediatr 1998;87:244-249.

4. Ellis KJ, Shypailo RJ, Hardin DS, Perez MD, Motil KJ, Wong WW, Abrams SA: Z score prediction model for assessment of bone mineral content in pediatric diseases. J Bone Miner Res 2001;16:1658-1656.

5. Molgaard C, Thomsen BL, Prentice A, Cole TJ, Michaelsen KF: Whole body bone mineral content in healthy children and adolescents. Arch Dis Child 1997;76:9-15.

6. Gennari L, Nuti R, Bilezikian JP: Aromatase activity and bone homeostasis in men. J Clin Endocrinol Metab 2004;89: 5898-5907.

7. Prior JC, Kirkland SA, Joseph L, Kreiger N, Murray TM, Hanley DA: Oral contraceptive use and bone mineral density in premenopausal women: cross-sectional, population-based data from the Canadian Multicentre Osteoporosis Study. CMAJ 2001;165:1023-1029.

8. Institute of Medicine, Food and Nutrition Board: Dietary Reference Intakes for Calcium, Phosphorus, Magnesium, Vitamin D, and Fluoride. Washington, National Academy Press, 1997.

9. Greer FR: Bone health: it's more than calcium intake. Pediatrics 2005;115:792-794.

10. Chevalley T, Bonjour JP, Ferrari, Hans ED, Rizzoli R: Skeletal site selectivity in the effects of calcium supplementation on areal bone mineral density gain: a randomized, double-blind, placebo-controlled trial in prepubertal boys. Clin Endocrinol Metab 2005;90:3342-3349.

11. Remsberg KE, Demerath EW, Schubert CM, Chumlea WC, Sun SS, Siervogel RM: Early menarche and the development of cardiovascular disease risk factors in adolescent girls: the Fels Longitudinal Study. J Clin Endocrinol Metab 2005;90: 2718-2724. 
